\section{Cuddling up in the dark}

\section{Michael P. Stryker}

IT is known that neural activity is involved in the courtship by which afferent nerve terminals establish relations with appropriate target cells in some parts of the nervous system. But whether the relevant activity is that of the presynaptic afferent, the postsynaptic cell or some harmony between the two has remained in question - most methods of interfering with activity perturb it on both sides. Now, on page 568 of this issue ${ }^{1}$, Hahm et al. provide compelling anatomical evidence that postsynaptic responses play a crucial role. This paper follows close behind another, in Science ${ }^{2}$, that reveals great order in the patterns of retinal activity occurring in development before vision is possible.

In most mammals with good binocular vision, the connections from the eyes to the main visual relay nucleus, the lateral geniculate nucleus (LGN), are arranged in layers that each receive input from one eye or the other. In a number of species, geniculate layers or sublaminae are also specific for the centre type ('On' or 'Off', responding to lightness or darkness, respectively) of the innervating retinal ganglion cells ${ }^{3}$.

At the level of the single cell, the specificity of connections is even greater: about half of the geniculate cells receive their effective excitatory input from only a single retinal ganglion cell; and among cells that receive several inputs, the inputs always originate from the same eye and from retinal ganglion cells of the same centre type ${ }^{4}$. Anatomical studies have revealed that single geniculate cells actually do manage this feat of ensuring that their hundreds of synaptic contacts are made with only one or a few of the hundreds of retinal afferents with which their dendritic fields overlap ${ }^{5}$.

How are these synaptic partners chosen with such precision? A most attractive hypothesis has been that discharge activity of retinal ganglion cells is locally correlated among cells of the same centre type within each small region of the retina, so that the timing of neural activity provides a high-resolution signal of the eye of origin, neighbourhood relationships and centre-type of each retinal terminal at every point within a target structure. If early synapses were hypothesized to have the hebbian property that they increased in strength according to the correlation between pre- and postsynaptic activity, then such input activity could lead initially diffuse projections to refine into precise maps, layers and single inputs ${ }^{6}$.

In normal development, retinal inputs from the two eyes initially innervate the LGN without regard to layer. Later (during the last few weeks of fetal life in the cat) they lose connections to the inappropriate regions and vastly expand their synaptic terminal arbor (branching structure) in appropriate regions so as to form eye-specific layers?
Although there is no visual stimulation during this period of life, retinal ganglion cells are active ${ }^{8}$ - they seem to discharge rhythmically, in waves of activity that propagate at various orientations across the retina ${ }^{2}$. Blocking this pre-visual activity of the retinal nerve fibres (and probably also that of the geniculate cells) by infusions of tetrodotoxin into fetal brains prevents the eye-specific layers from forming9.

Hahm et al. ${ }^{1}$ have now found that, in normal development, retinal afferent terminal arbors are progressively confined to single geniculate sublaminae. Locally or systemically applying blockers of the $N$-methyl- $D$ aspartate (NMDA) subtype of glutamate receptor, which mediates much of the input to the geniculate cells, would be expected to have little or no effect on retinal afferent activity, but it prevents the normal rearrangement of the retinal arbors ${ }^{1}$. These new results are consistent with the operation of hebbian synapses in the normal development of retinogeniculate connections.

The NMDA receptor has attracted enormous interest, because its biophysical properties allow it to inject calcium into the postsynaptic cells specifically at synapses whose synaptic activation takes place in association with other activity that depolarizes the cell ${ }^{10}$. In the CA1 region of the hippocampus, calcium entry through NMDA receptors seems to be necessary, and perhaps sufficient, for the long-term potentiation model of adult synaptic plasticity ${ }^{11}$. The NMDA receptors may play a similarly crucial part in amphibian visual-system development ${ }^{12}$. In the mammalian visual system, both pre- and postsynaptic cells may be studied anatomically and physiologically in vivo and in vitro. Future studies of this system may reveal whether calcium entry through NMDA receptors, or some other consequence of harmonious pre- and postsynaptic activity, mediates the synaptic plasticity responsible for the development of precise synaptic connections.

Michael P. Stryker is in the Department of Physiology and the Neuroscience Graduate Program, University of California, San Francisco, California 94143-0444, USA.

\footnotetext{
1. Hahm, J.-O., Langdon, R. B. \& Sur, M. Nature 351 568-570 (1991)

2. Meister, M. et al. Science 252, 939-943 (1991).

3. Stryker, M. P. \& Zahs, K. R. J. Neurosci. 3, 1943-1951 (1983).

4. Mastronarde, D. N. J. Neurophysiol. 57, 357-413 (1987).

5. Hamos, J. E. et al. J. comp. Neuro. 259, 165-192(1987).

6. Stent, G. S. Proc. natn. Acad. Sci. U.S.A. 70, 997-1001 (1973).

7. Shatz, C. J. J. Neurobiol. 21, 197-211 (1990).

8. Galli, L. \& Mafei L. Science 242, 90-91 (1988)

9. Sretavan, D. W., Shatz, C. J. \& Stryker, M. P. Nature 336, 468-471 (1988).

10. Nowak, L. et al. Nature 307, 462-465 (1984)

11. Malenka, R. C. et al. Science 242, 81-84 (1988).

12. Constantine-Paton, M. et al. A. Rev. Neurosci. 13, 129-154 (1990).
}

\section{Ghostly graphite}

CARBon fibre is made by carbonizing a carefully tensioned polymeric fibre. The tension aligns the growing graphite molecules in their 'strong' direction, giving the resulting fibre great strength and stiffness. But graphite is essentially two-dimensional. An oriented carbon-sheet product should be stronger and stiffer still. So Daedalus is inventing one.

His first idea was simply to carbonize acrylic sheeting while stretching it in two dimensions. But he now plans to start from a foamed polymer such as polyurethane. DREADCO's chemical engineers are polymerizing and foaming very dilute solutions of such materials. The resulting products resemble ultra-fine soap-foam. As the solvent evaporates, the cell walls of the foam thin to the desired thickness.

To graphitize this material evenly, Daedalus heats it in a big microwave oven. Pyrolysis-gas and residual solvent vapour build up in the closed foam-cells, and each tiny film-facet as it chars is tensioned and oriented by the resulting expansion. As in all liquid-foam structures, these individual facets all meet at $120^{\circ}$. At this angle, graphite sheets should combine very firmly, in a sort of polymeric extension of the triptycene molecule. When the process has been properly optimized, the resulting 'foamed graphite' should be immensely strong and rigid for its weight.

DREADCO's foamed graphite will be available in many grades. Thick foams will give dense materials resembling mattblack wood, ideal for load-bearing components such as beams and planks. More attenuated foams, formed as thin sheets and faced with polymer film, will compete with aluminium sheet in stressed-skin structures such as aircraft and pressurepiping. The thinnest foam of all will give an open network of monolayer graphite. This tenuous and ghostly product, a sort of polymeric higher fullerene, will have a low bulk density and will be very difficult to see. Like that other expanded solid, silica aerogel, it will be mainly empty space. Even so, it will have surprising strength and rigidity, amazing thermal insulation properties and will conduct electricity in strange ways. Like activated charcoal, it should also absorb gas readily.

If that gas is oxygen, of course, the result will be combustible or even explosive. The thicker, structural varieties of foamed graphite should take up gas too slowly to be hazardous from this effect. Even so, Daedalus is planning an autodestructive grade which, when exposed to pure oxygen, slowly acquires the explosive energydensity of dynamite. It should prove ideal for those fashionable architectural or technological monstrosities which, a few years later, turn out so horrific that they have to be destroyed.

David Jones 